\title{
Características de usuários frequentes de serviços de urgência: revisão integrativa
}

Characteristics of frequent emergency care service clients: an integrative review

Características de pacientes frecuentes de servicios de urgencia: revisión integrativa

Aline Marques Acosta ${ }^{1}$, Maria Alice Dias da Silva Lima²

${ }^{1}$ Enfermeira, Mestre em Enfermagem. Discente do Programa de Pós-Graduação em Enfermagem, nível Doutorado, da Escola de Enfermagem da Universidade Federal do Rio Grande do Sul (EENF/UFRGS). Porto Alegre, RS, Brasil. E-mail: aline.acosta@gmail.com.

${ }^{2}$ Enfermeira, Doutora em Enfermagem. Professora Associada IV da EENF/UFRGS. Porto Alegre, RS, Brasil. E-mail: malice@enf.ufrgs.br.

\section{RESUMO}

Este estudo teve como objetivo identificar e analisar a produção de conhecimento sobre as características dos usuários frequentes dos serviços de urgência. Realizou-se uma revisão integrativa, com busca nas bases de dados Science Direct, Cumulative Index to Nursing and Allied Health Literature (CINAHL), Medical Literature Analysis and Retrieval System Online (MEDLINE), Literatura Latino-Americana e do Caribe em Ciências da Saúde (LILACS) e Scientific Electronic Library Online (SCIELO). Foram selecionados 26 artigos que atenderam aos critérios de inclusão. Evidenciou-se que, de modo geral, usuários frequentes têm mais chances de ser do sexo feminino, ter idade entre 30 e 65 anos e ser desempregado. São geralmente socioeconomicamente mais vulneráveis e apresentam problemas de saúde precários, utilizando, dessa forma, frequentemente outros serviços de saúde. Destaca-se a falta de estudos sobre o tema no âmbito nacional, como também a escassez de literatura internacional com evidências fortes.

Descritores: Enfermagem em Emergência; Serviços Médicos de Emergência; Serviço Hospitalar de Emergência.

\section{ABSTRACT}

The objective of this study was to identify and analyze the knowledge development regarding the characteristics of frequent emergency care service clients. An integrative review was performed, with searches on the databases of the Science Direct, Cumulative Index to Nursing and Allied Health Literature (CINAHL), Medical Literature Analysis and Retrieval System Online (MEDLINE), Latin-American and Caribbean Health Sciences Literature (LILACS) and the Scientific Electronic Library Online (SCIELO). A total of 26 articles met the inclusion criteria and were, thus, selected. It was found that, generally, frequent clients are mostly female, of ages between 30 and 65 years and unemployed. They are generally more socioeconomically vulnerable and have more health problems, and, therefore, use other health systems more often. It is highlighted that studies on this theme are scarce in Brazil, as are international studies with strong evidence.

Descriptors: Emergency Nursing; Emergency Medical Services; Emergency Service, Hospital.

\section{RESUMEN}

El estudio objetivó identificar y analizar la producción de conocimiento sobre las características de pacientes frecuentes de los servicios de urgencia. Se realizó una revisión integrativa, buscando en las bases Science Direct, Cumulative Index to Nursing and Allied Health Literature (CINAHL), Medical Literature Analysis and Retrievel Sistem Online (MEDLINE), Literatura Latino Americana y del Caribe en Ciencias de la Salud (LILACS) y Scientific Electronic Library Online (SCIELO). Fueron seleccionados 26 artículos atendiendo los criterios de inclusión. Se demostró que, de manera general, el paciente frecuente es mayoritariamente de sexo femenino, con edad de 30 a 65 años, desempleado. Son, en general, más vulnerables a nivel socioeconómico y presentan problemas de salud precarios, utilizando de tal forma, con frecuencia, otros servicios de salud. Se destaca la falta de estudios sobre la temática en el ámbito nacional, así como también la escasez de literatura internacional con evidencias fuertes.

Descriptores: Enfermería de Urgencia; Servicios Médicos de Urgencia; Servicio de Urgencia en Hospital. 


\section{INTRODUÇÃO}

Os serviços de urgência constituem um importante componente da assistência à saúde. São serviços que têm como objetivo diminuir a morbimortalidade e as sequelas incapacitantes $^{(1)}$, oferecendo tratamento imediato e provisório a pessoas acometidas por agravos agudos de qualquer natureza(2). Contudo, identifica-se uma crescente demanda às unidades de urgência, tanto no cenário nacional quanto no internacional, que muitas vezes não corresponde ao caráter de urgência ${ }^{(3-4)}$.

Observa-se, ainda, que muitos usuários têm demonstrado uma persistente preferência na procura pelos serviços de urgências hospitalares, buscando repetitivamente atendimento nesses serviços. Indivíduos que procuram atendimento muitas vezes em serviços de urgência em determinado período de tempo são considerados usuários frequentes, também conhecidos como hiperutilizadores. Apesar de serem um pequeno grupo, comparado ao total de usuários que buscam atendimento, esses indivíduos são responsáveis por uma elevada porcentagem da demanda nesses serviços ${ }^{(5)}$. A utilização frequente dos serviços de urgência tem se tornado uma preocupação para gestores e profissionais da saúde, pois gera mais custos para o sistema de saúde, como também contribui para a sobrecarga e superlotação dos serviços envolvidos(5-6).

Embora as principais queixas e morbidades dos indivíduos que procuram o serviço de urgência repetidamente sejam bem conhecidas pelos profissionais de saúde, os usuários frequentes são muitas vezes estigmatizados pelos profissionais, que supõem que as suas queixas são inapropriadas para os serviços de urgência ${ }^{(7)}$. Porém, autores afirmam que este fato não reflete exatamente a natureza da utilização frequente dos serviços de urgência(5).

$\mathrm{Na}$ realidade brasileira, foram identificados como motivos para a frequente utilização de um serviço de urgência as dificuldades de acesso aos atendimentos nas unidades de atenção primária à saúde, representadas pela demora no atendimento e no agendamento das consultas, e os problemas na acessibilidade organizacional, devido aos turnos de funcionamento, tipo de marcação e horário das consultas( ${ }^{(8)}$.

De acordo com pesquisadores da temática, o melhor entendimento sobre as características dos usuários frequentes possibilita a identificação das necessidades de saúde e a elaboração de estratégias para qualificar a assistência e diminuir as reincidências nos serviços de urgência ${ }^{(6,9)}$, para se pensar em alternativas para reduzir a utilização frequente e a superlotação dos serviços de urgência(10-11).

No entanto, não existem pesquisas que tratem das características dos usuários frequentes no Brasil, justificando a necessidade de sintetizar evidências já publicadas na literatura internacional sobre o tema, visando qualificar a assistência e diminuir as reincidências nos serviços de urgência, como também guiar o desenvolvimento de futuras investigações. Portanto, realizou-se este estudo com o objetivo de identificar e analisar a produção de conhecimento sobre as características dos usuários frequentes dos serviços de urgência.

\section{METODOLOGIA}

Realizou-se uma revisão integrativa, método que sintetiza estudos publicados e possibilita conclusões gerais a respeito de uma temática, sintetizando o estado do conhecimento de um determinado assunto e demonstrando lacunas para a realização de novos estudos. Para o desenvolvimento desta revisão integrativa foram utilizadas cinco etapas: identificação do problema, busca na literatura, avaliação dos dados, análise dos dados e apresentação da revisão integrativa ou síntese do conhecimento(12).

Рага a identificação do problema, utilizou-se a pergunta norteadora: Qual é a produção de conhecimento sobre as características dos usuários frequentes dos serviços de urgência?

Os critérios de inclusão utilizados para estabelecer a amostra foram: artigos de pesquisa e revisões sistemáticas que abordam as características de usuários frequentes de serviços de urgência, publicados no período de janeiro de 2000 a maio de 2012, nos idiomas inglês, espanhol e português, com acesso on-line ao texto completo.

Foram utilizados os descritores controlados dos Descritores em Ciência da Saúde (DeCS) "Serviços Médicos de Emergência", "Serviço Hospitalar de Emergência" e "Enfermagem em Emergência" e as do Medical Subject Headings (MeSH) "Emergency Hospital Service", "Emergency Nursing" e "Emergency Medical Services". Para reduzir e restringir os vieses da busca de 
artigos foram acrescentados os descritores nãocontrolados "usuários frequentes", "hiperfrequentadores", "frequent flyers" e "heavy users".

A busca ocorreu em maio de 2012 e foram utilizadas as bases de dados Science Direct, Cumulative Índex to Nursing and Allied Health Literature (CINAHL), Medical Literature Analysis and Retrievel Sistem Online (MEDLINE), Literatura Latino-Americana e do Caribe em Ciências da Saúde (LILACS) e Scielo. Realizaram-se todas as combinações possíveis entre os descritores em cada base de dados, utilizando-se o conector booleano "AND". Assim, foram encontrados 434 artigos, sendo 314 da base Science Direct, 27 da CINAHL, 31 da MEDLINE, 62 da LILACS e nenhum da Scielo.

Primeiramente, pela leitura do título e resumo e pela aplicação dos critérios de inclusão, 43 artigos foram selecionados. No entanto, após a leitura detalhada do material, foram excluídas 17 publicações que não apresentavam resultados ou discussões diretamente relacionados à temática. Desta forma, a amostra final foi composta por 26 artigos científicos.

A fim de se ter mais precisão nos resultados obtidos, a busca de literatura foi realizada de forma independente por dois avaliadores, que utilizaram as mesmas bases de dados e termos. Ao final das buscas, não houve discordâncias nos resultados, logo não foi necessária a consulta de um terceiro avaliador.

Para a avaliação dos estudos, foi elaborado um roteiro de coleta de dados que contemplou informações sobre o artigo, como autores, título, periódico, palavraschave, objetivo, tipo de estudo, amostra, local, técnica de coleta de dados, resultados e conclusões. Ainda, os artigos foram classificados em relação ao nível de evidência. Nesta revisão foi empregado um sistema de classificação composto por sete níveis, sendo: Nível I evidências oriundas de revisões sistemáticas ou metaanálise de relevantes ensaios clínicos; Nível II - evidências derivadas de pelo menos um ensaio clínico randomizado controlado bem delineado; Nível III - ensaios clínicos bem delineados sem randomização; Nível IV - estudos de coorte e de caso-controle bem delineados; Nível V revisão sistemática de estudos descritivos e qualitativos; Nível VI - evidências derivadas de um único estudo descritivo ou qualitativo e Nível VII - opinião de autoridades ou relatório de comitês de especialistas. As evidências pertencentes aos níveis I e II são consideradas fortes, de III a V, as evidências são moderadas e VI e VII são evidências fracas ${ }^{(13)}$.

A análise de dados foi realizada em duas etapas. Na primeira, para descrever a caracterização dos artigos encontrados, foram realizados cálculos de frequência simples e relativa. Na segunda etapa, procedeu-se a leitura detalhada das publicações e se realizou a análise do conteúdo dos artigos.

A apresentação dos dados é mostrada em um quadro sinóptico que sumariza as informações obtidas neste estudo. Após, são apresentadas as principais características e o conteúdo dos artigos analisados.

\section{RESULTADOS E DISCUSSÃO}

Dos 26 artigos que compuseram a amostra, 92,3\% estavam no idioma inglês, um estudo (3,85\%) estava em português e outro (3,85\%) em espanhol. Com relação ao país de origem, 12 estudos (46,15\%) são provenientes dos Estados Unidos da América, três (11,53\%) do Canadá, três $(11,53 \%)$ da Austrália, dois (7,7\%) da Grã-Bretanha e cada um dos demais são de países distintos, sendo China, Espanha, Irlanda, Portugal, Singapura e Suécia.

Entre os anos 2000 e 2007 foram publicados 11 artigos (42,3\%), sendo que se teve uma média anual de 1,4 artigos produzidos por ano nesse período. Foi observado um aumento no número de publicações a partir de 2008 , de forma que $57,7 \%$ dos trabalhos foram publicados no período entre 2008 e 2012. Esse último período teve uma média de três artigos por ano, o que é mais que o dobro da média anterior. Esse fato demonstra que a preocupação com os usuários frequentes tem aumentado nos últimos anos, o que se justifica pelo aumento da demanda dos serviços de urgência nas últimas décadas e da consequente superlotação(14).

Quanto ao nível de evidência, 23,77\% dos artigos foram classificados como evidências moderadas e $69,23 \%$, fracas. Os estudos foram desenvolvidos com diferentes métodos, porém se destacam as pesquisas quantitativas retrospectivas e transversais, que descrevem as principais características dos usuários frequentes, comparando-os com os demais usuários do serviço de urgência. Os estudos quantitativos permitem conhecer e comparar características de indivíduos, identificando fatores relacionados à utilização frequente dos serviços de urgência. Contudo, não é aprofundada a perspectiva dos usuários, seus valores e significados que 
podem induzi-los a procurar atendimento repetidamente, informações que apenas em estudos qualitativos se poderiam obter ${ }^{(15)}$. O Quadro 1 apresenta os artigos incluídos, indicando o nível de evidência e os principais resultados.

Quadro 1: Distribuição dos artigos, segundo autores, título e fonte, Porto Alegre, RS, 2010.

\begin{tabular}{|c|c|c|}
\hline $\begin{array}{c}\text { Autores, periódico e } \\
\text { ano }\end{array}$ & $\begin{array}{l}\text { Nível de } \\
\text { evidência }\end{array}$ & Resultados \\
\hline $\begin{array}{c}\text { Doup et al. Ann } \\
\text { Emerg Med. 2010(16) }\end{array}$ & VI & $\begin{array}{l}\text { Usuários frequentes eram responsáveis por } 9,9 \% \text { dos atendimentos no } \\
\text { serviço. Comparados aos usuários eventuais, utilizavam mais a atenção } \\
\text { primária e secundária. }\end{array}$ \\
\hline $\begin{array}{l}\text { Wajnberg et al. J } \\
\text { Emerg Med. 2011(17) }\end{array}$ & VI & $\begin{array}{c}\text { A maioria dos idosos que são usuários frequentes foi considerada menos } \\
\text { urgente na classificação de risco e relatou ter uma fonte habitual de } \\
\text { cuidado. }\end{array}$ \\
\hline $\begin{array}{l}\text { Kirby et AL. BMC } \\
\text { Health Serv Res. } \\
\qquad 2010^{(18)}\end{array}$ & VI & $\begin{array}{c}\text { Usuários frequentes eram mais propensos a ser mais velhos e serem } \\
\text { classificados como urgentes. As condições crônicas foram fortemente } \\
\text { associadas com reinternações frequentes. }\end{array}$ \\
\hline $\begin{array}{l}\text { Paul et al. Emerg } \\
\text { Med J. 2010(19) }\end{array}$ & IV & $\begin{array}{l}\text { Fatores significativamente associados à utilização frequente do serviço de } \\
\text { urgência foram idade superior a } 75 \text { anos, sexo masculino e busca por } \\
\text { atendimento das } 16 \text { horas à meia noite. }\end{array}$ \\
\hline $\begin{array}{l}\text { Tangherline et al. } \\
\text { Prehosp Disaster } \\
\text { Med. } 2010^{(20)}\end{array}$ & IV & $\begin{array}{l}\text { Ser homem, morador de rua e ter problemas cardiovascular, pulmonar e } \\
\text { alcoolismo foram associados à maior utilização do serviço pré-hospitalar } \\
\text { móvel de urgência por idosos. Não foram encontradas diferenças } \\
\text { significativas com relação ao uso de serviços primários de saúde. }\end{array}$ \\
\hline $\begin{array}{l}\text { LaCalle, Rabin. Ann } \\
\text { Emerg Med. } 2010^{(5)}\end{array}$ & V & $\begin{array}{l}\text { Usuários frequentes eram mais doentes do que os demais usuários e } \\
\text { tinham mais chances de uma internação hospitalar. Esses indivíduos } \\
\text { utilizavam mais vezes outros serviços de saúde. }\end{array}$ \\
\hline $\begin{array}{l}\text { Sandoval, et al. J } \\
\text { Emerg Med. 2010(21) }\end{array}$ & VI & $\begin{array}{l}\text { Hipertensão, anemia falciforme e depressão eram mais prevalentes em } \\
\text { usuários frequentes. Eles tinham pior estado de saúde geral, elevado nível } \\
\text { de estresse e baixo apoio social. }\end{array}$ \\
\hline $\begin{array}{l}\text { Moore et al. Emerg } \\
\text { Med J. 2009(22) }\end{array}$ & VI & $\begin{array}{c}\text { Diferenças no perfil dos usuários apareceram a partir da quarta reindiência } \\
\text { no ano. Usuários frequentes eram mais velhos e classificados com riscos } \\
\text { mais elevados do que usuários eventuais. }\end{array}$ \\
\hline $\begin{array}{l}\text { Aisiku, et al. Ann } \\
\text { Emerg Med. 2009(23) }\end{array}$ & IV & $\begin{array}{l}\text { Usuários frequentes com anemia falciforme tinham níveis mais baixos de } \\
\text { hematócritos, realizavam mais transfusões e apresentavam mais crises e } \\
\text { dor do que usuários eventuais. }\end{array}$ \\
\hline $\begin{array}{c}\text { Milbrett, Halm. J } \\
\text { Emerg Nurs. 2009(24) }\end{array}$ & VI & $\begin{array}{c}\text { Usuários frequentes eram mais comumente do sexo feminino, solteiros e } \\
\text { desempregados. A maioria tinha história médica de doença crônica e } \\
\text { consultava com médico na atenção primária. }\end{array}$ \\
\hline $\begin{array}{l}\text { Shiber, Longley, } \\
\text { Brewer. Am J Emerg } \\
\text { Med. 2009(6) }\end{array}$ & IV & $\begin{array}{c}\text { O grupo de usuários frequentes era mais velho e apresentava mais doenças } \\
\text { cardiovasculares, geniturinárias e psiquiátricas. O acesso aos serviços de } \\
\text { saúde em geral era similar a demais população que busca assistência no } \\
\text { serviço de urgência, assim como os níveis de hospitalização. }\end{array}$ \\
\hline $\begin{array}{l}\text { Huang, et al. J } \\
\text { Formos Med Assoc. } \\
2008^{(25)}\end{array}$ & VI & $\begin{array}{c}\text { Quanto maior for o número de reincidências no serviço de urgência, maior } \\
\text { era a chance de o usuário utilizar a atenção primária e serviços hospitalares } \\
\text { ambulatoriais. }\end{array}$ \\
\hline $\begin{array}{l}\text { Jelinek, et al. Med J } \\
\text { Aust. } 2008^{(26)}\end{array}$ & VI & $\begin{array}{l}\text { Usuários frequentes tinham mais problemas relacionados a doenças } \\
\text { psiquiátricas e circulatórias. Apresentavam condições mais urgentes e } \\
\text { maiores índices de admissão que os demais pacientes. }\end{array}$ \\
\hline $\begin{array}{l}\text { Mehl-Madrona. Can J } \\
\text { Rural Med.2008(27) }\end{array}$ & VI & $\begin{array}{c}\text { 93\% dos usuários frequentes tinham pelo menos uma doença psiquiátrica. } \\
\text { Eles frustravam os profissionais da saúde, que achavam que as queixas } \\
\text { eram inapropriadas para o serviço. }\end{array}$ \\
\hline $\begin{array}{l}\text { Oliveira. Acta Med } \\
\text { Port. } 2008^{(28)}\end{array}$ & IV & $\begin{array}{l}\text { Os fatores associados com a utilização frequente do serviço de urgência } \\
\text { foram: ser idoso, ter um problema clínico e ter uma internação hospitalar. }\end{array}$ \\
\hline $\begin{array}{l}\text { Fuda, Immekus. Ann } \\
\text { Emerg Med. } 2006^{(29)}\end{array}$ & VI & $\begin{array}{c}\text { O status de usuário frequente é temporário na maioria das vezes. A } \\
\text { procura de usuários frequentes pelos serviços de urgência é tão apropriada } \\
\text { quanto a dos usuários não frequentes. }\end{array}$ \\
\hline
\end{tabular}




\begin{tabular}{|c|c|c|}
\hline $\begin{array}{l}\text { Autores, periódico e } \\
\text { ano }\end{array}$ & $\begin{array}{l}\text { Nível de } \\
\text { evidência }\end{array}$ & Resultados \\
\hline $\begin{array}{l}\text { Hunt, et al. Ann } \\
\text { Emerg Med. } 2006^{(9)}\end{array}$ & VI & $\begin{array}{c}\text { Usuários frequentes utilizavam mais outros serviços de saúde do que os } \\
\text { demais usuários, no entanto, apresentavam problemas de saúde mais } \\
\text { precários. }\end{array}$ \\
\hline $\begin{array}{l}\text { Blank, et al. J Emerg } \\
\quad \text { Nurs, 2005(30) }\end{array}$ & VI & $\begin{array}{c}\text { Acesso aos serviços de saúde é melhor para usuários que utilizaram o } \\
\text { serviço de urgência mais de } 12 \text { vezes ao ano do que àqueles que usaram de } \\
4 \text { a } 11 \text { vezes. }\end{array}$ \\
\hline $\begin{array}{l}\text { Torrecillas et al. } \\
\text { Emergencias. 2004(31) }\end{array}$ & VI & $\begin{array}{l}\text { As características de usuários frequentes variam conforme a idade e o } \\
\text { sexo. Os grupos mais representativos eram meninos e mulheres na idade } \\
\text { fértil. }\end{array}$ \\
\hline $\begin{array}{l}\text { Chan, Ovens. Ann } \\
\text { Emerg Med. 2004(32) }\end{array}$ & VI & $\begin{array}{l}\text { Pessoas com enxaqueca crônica são um grupo de usuários frequentes dos } \\
\text { serviços de urgência, que utilizam o dobro de vezes a atenção primária do } \\
\text { que os demais usuários frequentes. }\end{array}$ \\
\hline $\begin{array}{l}\text { Dent et al. Emerg } \\
\text { Med (Fremantle). } \\
\qquad 2003^{(33)}\end{array}$ & VI & $\begin{array}{c}\text { Os } 500 \text { usuários mais frequentes de um serviço de urgência tinham } \\
\text { problemas de saúde considerados adequados para o serviço e } \\
\text { apresentaram uma alta taxa de mortalidade. }\end{array}$ \\
\hline $\begin{array}{l}\text { Byrne, et al. Ann } \\
\text { Emerg Med. } 2003^{(34)}\end{array}$ & VI & $\begin{array}{l}\text { Usuários frequentes também utilizavam muito outros serviços de saúde. } \\
\text { Eles eram economicamente desprovidos e tinham pior saúde mental e } \\
\text { baixo apoio social. }\end{array}$ \\
\hline $\begin{array}{l}\text { Chan, Ovens. Can } \\
\text { Fam Physician. } \\
2002^{(35)}\end{array}$ & VI & $\begin{array}{c}\text { Maioria dos usuários frequentes consulta periodicamente com um médico } \\
\text { na atenção primária e eram mais referenciados a especialistas por médicos } \\
\text { da atenção básica. }\end{array}$ \\
\hline $\begin{array}{l}\text { Hansagi, et al. Ann } \\
\text { Emerg Med. } 2001^{(36)}\end{array}$ & VI & $\begin{array}{l}\text { A maioria dos usuários frequentes utilizava a atenção primária, porém 13\% } \\
\text { usavam o serviço de urgência como única fonte de cuidado. Usuários } \\
\text { frequentes tinham maiores percentuais de internação hospitalar e de } \\
\text { mortalidade. }\end{array}$ \\
\hline $\begin{array}{l}\text { Williams, et al. J } \\
\text { Psychosom Res. } \\
\qquad 2001^{(37)}\end{array}$ & IV & $\begin{array}{l}\text { Usuários frequentes eram mais velhos e tinham pior estado de saúde do } \\
\text { que os demais. Mais de } 90 \% \text { relataram ter história de doença crônica. }\end{array}$ \\
\hline $\begin{array}{l}\text { Mandelberg, Kuhn, } \\
\text { Kohn. Acad Emerg } \\
\text { Med, 2000(38) }\end{array}$ & IV & $\begin{array}{c}\text { Usuários frequentes tinham mais exarcebações de doenças crônicas e } \\
\text { problemas relacionados ao álcool que os usuários eventuais. Somente 38\% } \\
\text { dos usuários frequentes permaneceram utilizando o serviço de urgência } \\
\text { repetidas vezes por mais um ano. }\end{array}$ \\
\hline
\end{tabular}

Dentre os periódicos em que os artigos foram publicados, o Annals of Emergency Medicine se destacou com oito estudos (30,77\%), seguido por Journal of Emergency Medicine, Emergency Medicine Journal e Journal of Emergency Nursing, cada um com duas publicações $(7,69 \%)$. Outros periódicos tiveram apenas uma publicação. Identificou-se que a grande maioria dos estudos foram publicados em periódicos da área da medicina e apenas dois eram da Enfermagem.

No que concerne à definição de usuário frequente, foi possível identificar que, mesmo sendo objeto de estudo de diversas pesquisas, ainda não há um mínimo de frequência esperada para definir usuários frequentes e eventuais. O limiar utilizado nos estudos analisados variou de três a 12 visitas ao serviço de urgência por ano.

O limite mais usado para definir usuário frequente foi de quatro ou mais atendimentos no serviço de urgência em um ano (12 meses). A maioria dos autores de artigos que utilizaram esta definição relatou que a escolheu por ser a mais utilizada(25,28,34,36). Um dos estudos usou o parâmetro de quatro ou mais visitas ao ano após avaliar o impacto de usuários que utilizaram o serviço de uma a sete vezes ou mais. Constatou que quem utilizou no mínimo quatro vezes era responsável por mais de $25 \%$ da demanda, número considerado pelos autores impactante para um pequeno grupo de indivíduos ${ }^{(9)}$. Em concordância com esse estudo, autores de uma pesquisa realizada na Grã-Bretanha definiram que as pessoas que procuram atendimento no serviço mais de quatro vezes ao ano são consideradas frequentes, pois apresentam necessidades de saúde diferentes dos usuários eventuais, como também representam um grupo mais vulnerável a $\operatorname{riscos}^{(39)}$.

Com relação à prevalência dos usuários frequentes nos serviços de urgência, identificou-se que existe uma diferença nos resultados dos estudos analisados. Enquanto alguns mostram que estes usuários são em pequeno número comparado com todos os pacientes do 
serviço, variando de $0,3 \%$ a $4 \%(25-26,29,32,34,36,38)$, outros indicam um número mais elevado, correspondendo de $6 \%$ a $9,7 \%(9,16,19,21,28,30)$. No Brasil, um estudo publicado em 2011, que foi realizado em um serviço de urgência em Pelotas/RS, identificou que quase $20 \%$ dos indivíduos que buscaram atendimento tinham utilizado o serviço três ou mais vezes em um ano e 5,9\% consultaram três ou mais vezes em três meses ${ }^{(2)}$.

Nos trabalhos analisados, foram abordadas porcentagens referentes ao número de atendimentos realizados com usuários frequentes em relação ao total de atendimentos dos serviços de urgência, demonstrando preocupação dos autores na grande demanda que estes indivíduos geram aos serviços. Usuários que utilizaram três vezes ou mais ao ano eram responsáveis por $13 \%$ a $33 \%$ da procura pelos serviços de urgência $(9,16,21,25,28$ $30,36,38)$, sendo que quando analisados aqueles que usaram no mínimo 12 vezes, a porcentagem baixava para 3,5\% ${ }^{(32)}$. Um estudo realizado em Massachusetts, Estados Unidos, identificou que apenas $1 \%$ dos residentes da cidade utilizou a urgência hospitalar mais de cinco vezes, mas condizia a $17,6 \%$ da demanda do serviço ${ }^{(29)}$.

O que intriga ainda mais os estudiosos é o fato da utilização frequente estar aumentando, tanto em relação à quantidade de usuários, como em relação à frequência de busca de atendimento. Nos Estados Unidos, foram analisadas as mudanças no perfil das pessoas que utilizaram os serviços de urgência entre 1996 e 2005, constatando que está aumentando a quantidade de usuários frequentes ${ }^{(14)}$. Estudo realizado em Portugal notou que um único indivíduo frequentou a urgência hospitalar 401 vezes em um ano(28). Já em Perth, Austrália, autores identificaram que um usuário buscou atendimento 218 vezes no ano de 2006, número inferior às máximas dos anos anteriores, indicando que o número máximo de reincidências nos serviços de urgência está aumentando também ${ }^{(26)}$.

A partir das implicações avaliadas pelos autores, alguns pesquisadores justificam que a utilização frequente é uma das causas da superlotação. Uma revisão sistemática indicou que fatores de entrada nos serviços de urgência estão relacionados à crescente demanda, na qual usuários frequentes a afetam diretamente ${ }^{(4)}$. Contudo, em um estudo realizado em Washington, EUA, usuários altamente frequentes do serviço de urgência, que o utilizaram mais de 20 vezes ao ano, provocavam um impacto mínimo na superlotação do serviço, já que esses indivíduos permaneciam menos tempo no serviço(40).

A utilização frequente dos serviços de saúde pode ser influenciada por fatores demográficos de sexo e idade. Com relação ao sexo, os estudos tiveram resultados diferentes. Dos 16 artigos que citaram essa característica, seis relataram que as mulheres eram em maior quantidade entre os usuários frequentes ${ }^{(9,17,24,28-29,31,36)}$. Contudo, outros quatro trabalhos referiram que não havia diferença significativa entre os dois $\operatorname{sexos}^{(6,25,30,34)}$ e, ainda, alguns estudos identificaram que homens eram em maior número(21-22,26,37-38).

Quase todos os trabalhos em que foi descrita a idade dos usuários identificaram que os frequentes são mais velhos do que os eventuais(6,18,21-22,24-26,28-30,32,34,36-38). Outros trabalhos evidenciaram que quanto maior o grupo de idosos (65 anos ou mais) atendidos no serviço, mais usuários frequentes existiriam ${ }^{(25,36)}$. Da mesma forma, uma revisão de literatura descreveu que pessoas dos grupos etários mais velhos são associadas à maior chance de ser usuário frequente, se comparados aos grupos mais $\operatorname{novos}^{(10)}$.

Embora os usuários frequentes sejam mais velhos do que os eventuais, os estudos mostram que eles têm idade que varia de 30 a $65 \operatorname{anos}^{(6,9,21,24,26,30,34,37-38)}$. Resultado similar foi encontrado em pesquisa que caracterizou os agravos clínicos atendidos pelo Serviço de Atendimento Móvel de Urgência (SAMU) de Porto Alegre-RS, na qual se evidenciou que a faixa etária que mais concentrou atendimentos foi a de 41 a 60 anos (cerca de $25 \%)^{(41)}$. Isso indica que uma população economicamente ativa tem necessitado de cuidados de urgência, e no caso de usuários frequentes, repetidamente, o que pode trazer prejuízos no estilo de vida, no cuidado da família e no trabalho.

Corroborando com isso, estudos indicam que a maioria dos usuários frequentes não está empregada ${ }^{(21,24,30)}$. Autores reconhecem que $78 \%^{(24)}$ a $88 \%{ }^{(30)}$ dessas pessoas são desempregadas, jovens ou aposentadas.

Poucos artigos analisaram aspectos socioeconômicos desses indivíduos. Algumas pesquisas internacionais mostram que a maioria dos usuários frequentes é coberta por algum tipo de plano de saúde, porém a grande parte é financiada pelo governo de uma forma ou de outra, o que indica que usuários frequentes são vulneráveis 
economicamente $\mathrm{f}^{(6,9,21,24,27,29-30,32,34)}$. O nível de pobreza desses usuários foi descrito em apenas um artigo ${ }^{(9)}$. Esse nível avalia a renda familiar anual e calcula, em cada país, o quanto gasta com alimentação, moradia e saúde. Nesse estudo, realizado nos Estados Unidos, evidenciou-se que $33 \%$ desses indivíduos dos serviços de urgência tinham renda familiar abaixo do nível de pobreza. Outra pesquisa evidenciou que existe associação significativa entre a falta de habitação e a utilização frequente por pessoas idosas $^{(19)}$.

Com relação às características clínicas de usuários frequentes, obtiveram-se resultados similares nos trabalhos analisados. A agudização de doenças crônicas pareceu ser um fator bem importante para a utilização repetida dos serviços de urgência. Conforme algumas pesquisas, essa é a principal queixa para a procura frequente dos serviços ${ }^{(6,19,24,34,36-38)}$.

As condições crônicas, por permanecerem um tempo prolongado ou indeterminado na vida das pessoas, as predispõem a fases de exacerbação e remissão de sinais e sintomas $^{(42)}$. Isso significa que nem sempre o indivíduo se sente doente, mas mesmo assim necessita de cuidados da família e de um profissional de saúde. Usuários frequentes parecem precisar de assistência continuada, com acompanhamento de um profissional de saúde e/ou serviço de saúde, e também de assistência de urgência, geralmente por agudização de suas doenças crônicas.

As comorbidades mais frequentemente encontradas nesses usuários eram relacionadas a problemas respiratórios, neurológicos, psiquiátricos, circulatórios, endócrinos e gastrointestinais(6,17,24,34,36-38). Em menor escala estavam os problemas musculoesqueléticos ${ }^{(6,34)}$ e renais(38).

Entre os problemas respiratórios, a asma se destacou, correspondendo de $4 \%$ a $20 \%$ da procura por usuários frequentes $(24,36,38)$. Dentre os problemas neurológicos está a convulsão/epilepsia(29). A anemia falciforme aparece como um problema circulatório mais comum nos usuários frequentes do que nos usuários eventuais(38), sendo que os usuários frequentes necessitam de transfusão sanguínea mais vezes(23).

Os problemas psiquiátricos são encontrados de $11 \%$ a $36 \%$ em usuários frequentes(24,34). Diagnósticos de saúde mental mais encontrados são: depressão e ansiedade ${ }^{(16,27)}$, distúrbios somatoformes, distimia, personalidade borderline e somatização(27). Alguns estudos descrevem que o abuso/dependência de álcool e drogas pode estar relacionado à utilização frequente dos serviços de urgência(5,16,20,26-27). Um estudo realizado na Irlanda identificou que $38 \%$ dos usuários frequentes eram mais comumente vistos com problemas relacionados ao álcool(34). Porém, algumas pesquisas obtiveram resultados diferenciados, em que a porcentagem desses indivíduos com problemas relacionados ao álcool/drogas era baixa, entre $3 \%$ e $4 \%(24,30,36)$.

Outro problema que foi bastante encontrado nesses indivíduos é a dor ${ }^{(24,27,30,32)}$. Problemas relacionados à dor correspondem a $27 \%$ da procura dos usuários frequentes. Os tipos de dor mais frequentes encontrados foram: enxaqueca e cefaléia, dor abdominal, mialgias/neuralgias e dores articulares ${ }^{(30)}$.

Autores atestam que usuários frequentes têm pior estado de saúde, além de grandes dificuldades para atividades físicas rotineiras e níveis maiores de estresse, se comparados aos usuários eventuais(21). Além disso, apresentam maiores taxas de mortalidade e maior probabilidade de obterem uma internação hospitalar do que os usuários eventuais ${ }^{(29)}$. Concordando com isso, um estudo qualitativo identificou que usuários frequentes percebem que seu problema de saúde é uma ameaça a sua vida ou a sua autonomia e, por isso, na percepção deles, necessitam de atendimento imediato(15). Pesquisas complementam esses dados indicando que usuários frequentes são classificados com maior gravidade nos protocolos de classificação de risco mais vezes do que os usuários eventuais ${ }^{(18,22)}$.

Tais achados indicam que os usuários frequentes têm saúde mais precária se comparados aos demais usuários e, então, espera-se que eles utilizem mais vezes os serviços de urgência. Porém, receia-se que esses serviços tenham se tornado a única fonte de cuidado para alguns usuários que se apresentam repetidamente no serviço, uma consequência da dificuldade de acesso aos demais serviços da rede assistencial.

Um estudo identificou que usuários altamente frequentes (aqueles que buscaram atendimento de urgência mais de 18 vezes em um ano) utilizam menos outros serviços de saúde do que usuários moderadamente frequentes (de sete a 17 visitas em um ano), o que sugere que os altamente frequentes utilizam os serviços de urgência em vez de outros serviços de saúde ${ }^{(16)}$. Outra pesquisa realizada no interior de São 
Paulo constatou que a maioria de usuários frequentes de um serviço de urgência não utilizava outros serviços de saúde, de forma que o serviço de urgência era o local onde eles faziam o acompanhamento da sua saúde. Isso implica em questões que vão além da demanda excessiva nos serviços de urgência, como o atendimento paliativo e pontual, com assistência fragmentada e descontínua. $O$ principal motivo da busca frequente por atendimento em serviços de urgência é a demora no agendamento das consultas, como também a dificuldade em obter medicações na atenção primária( ${ }^{(8)}$.

Da mesma forma, no Estado de Minas Gerais, usuários relataram ter dificuldade no agendamento de consulta, demora na obtenção de atendimento e falta de médicos na atenção primária, o que de fato contribui para a procura pelos serviços de urgência ${ }^{(3)}$. Conforme os autores, para o usuário, o tempo de espera nos serviços de urgência não importa tanto quando existe a certeza do atendimento, o que se contrapõe à sensação de contingência ou incerteza dos serviços da atenção primária. Um outro estudo evidenciou que serviços de urgência são algumas vezes uma alternativa, na percepção dos usuários, para atendimento mais fácil e rápido para as necessidades de saúde ${ }^{(43)}$.

Algumas pesquisas internacionais analisadas nesta revisão mostraram que indivíduos que utilizam muitas vezes os serviços de urgência são usuários frequentes em outros serviços de saúde ${ }^{(5,17,26)}$ e são mais prováveis de ter um acompanhamento de um médico na atenção primária do que usuários eventuais ${ }^{(21,32)}$. Uma pesquisa identificou que $72 \%$ dos usuários frequentes realizaram visitas a médicos na atenção primária, já visitas a departamentos ambulatoriais hospitalares corresponderam a 59\%(36). Além disso, usuários frequentes utilizam mais ambulatórios hospitalares(17), serviços públicos de Enfermagem, de bem-estar comunitário, de trabalho social e serviços psiquiátricos(34).

Corroborando com isso, autores de uma revisão de literatura afirmam que é claro que muitos usuários frequentes não obtêm cuidados de saúde em serviços de urgência exclusivamente ${ }^{(10)}$. Pode-se entender que o fato de usuários frequentes serem mais vulneráveis socioeconomicamente e mais doentes do que os usuários eventuais, proporciona que eles utilizem os serviços de urgência mais vezes do que os demais usuários, assim como os outros serviços de saúde.

\section{CONSIDERAÇÕES FINAIS}

Este estudo permitiu sintetizar os achados de publicações internacionais veiculadas nos periódicos da área da saúde entre os anos 2000 e 2012, relacionadas às características dos usuários frequentes de serviços de urgência e indicar as lacunas do conhecimento. Porém, é importante ressaltar que existem limitações para analisar as características de usuários, visto que os contextos em que os indivíduos estão inseridos são diferentes. Ainda, o fato de terem sido utilizadas apenas publicações com acesso on-line limitou a abrangência da pesquisa.

Os artigos incluídos nesta revisão apresentaram níveis de evidência considerados moderados e fracos. Isso indica uma escassez de pesquisas como revisões sistemáticas ou meta-análise de relevantes ensaios clínicos e ensaios clínicos randomizados bem delineados que iriam proporcionar evidências fortes sobre os usuários frequentes dos serviços de urgência.

Constatou-se que os autores são, majoritariamente, dos Estados Unidos da América, demonstrando o grande empenho dos pesquisadores desse país em estudar a utilização frequente. No Brasil, no entanto, não foi encontrada nenhuma publicação, mesmo sendo um tema que gera discussões entre gestores e profissionais da saúde.

Identificou-se que usuários frequentes constituem um grupo heterogêneo, com características que podem variar conforme idade, sexo e frequência de atendimento. Contudo, esses indivíduos apresentam problemas de saúde precários e suas queixas geralmente são adequadas para atendimento nos serviços de urgência.

Destaca-se a necessidade de implementação de programas em serviços de urgência que identifiquem usuários que estão em risco de buscar repetidamente atendimento, a fim de traçar as necessidades de saúde de cada usuário e desenvolver planos individuais de atendimento.

A utilização frequente de serviços de urgência é fortemente influenciada por fatores socioeconômicos e pelas doenças crônicas. Isso indica a importância da atuação da rede de atenção à saúde no cuidado a esses indivíduos, que necessitam de atendimento tanto de ações continuadas para a prevenção de agravos, quanto de pronto atendimento nas situações de agudização. A atenção multidisciplinar contínua e qualificada na rede assistencial pode contribuir para a diminuição da 
utilização frequente dos serviços de urgência. Nessa perspectiva, é essencial a comunicação entre os serviços de urgência e de atenção primária e secundária, com a formação e pactuação de fluxos de referência e contrareferência, para que o usuário frequente tenha cuidado contínuo.

Recomenda-se a realização de estudos sobre os motivos da utilização frequente dos serviços de urgência e pesquisas relacionadas ao acesso e utilização de outros

\section{REFERÊNCIAS}

1. Azevedo ALCS, Pereira AP, Lemos C, Coelho MF, Chaves LDP. Organização de serviços de emergência hospitalar: uma revisão integrativa de pesquisa. Rev. Eletr. Enf. [Internet]. 2010 [cited 2012 feb 2];12(4):736-45. Available from:

http://www.fen.ufg.br/revista/v12/n4/v12n4a20.htm 2. Carret MLV, Fassa AG, Paniz VMV, Soares PC. Características da demanda do serviço de saúde de emergência no Sul do Brasil. Cien Saude Colet. 2011;16(supl 1):1069-79.

3. Oliveira LH, Mattos RA, Souza Al. S. Cidadãos peregrinos: os "usuários" do SUS e os significados de sua demanda a prontossocorros e hospitais no contexto de um processo de reorientação do modelo assistencial. Cien Saude Colet. 2009;14(5):1929-38.

4. Hoot NR, Aronsky D. Systematic Review of Emergency Department Crowding: causes, effects and solutions. Ann Emerg Med. 2008:52(2):126-36.

5. LaCalle E, Rabin E. Frequent Users of Emergency

Departments: The Myths, the Data, and the Policy Implications. Ann Emerg Med. 2010;56(1):42-8.

6. Shiber JR, Longley MB, Brewer KL. Hyper-use of the ED. Am J Emerg Med. 2009;27(5):588-94.

7. Acosta AM, Pelegrini AHW, Lima MADS. Percepção dos profissionais de saúde sobre os usuários frequentes dos serviços de urgência e emergência: revisão integrativa. Enferm Foco. 2011;2(2):141-4.

8. Souza MF, Figueiredo LA, Pinto IC. Análise da utilização do serviço de pronto-socorro na percepção do usuário. Cienc Cuid Saude. 2010; 9(1):13-20.

9. Hunt KA, Weber EJ, Showstack JA, Colby DC, Callaham ML. Characteristics of Frequent Users of Emergency Departments. Ann Emerg Med. 2006;48(1):1-8.

10.Pines JM, Asplin BR, Kaji AH, Lowe RA, Magid DJ, Raven M, et al. Frequent user of emergency department services: gaps in knowledge and a proposed research agenda. Acad Emerg Med. 2011;18(6):64-9.

11. Okin RL, Boccellari A, Azocar F, Shumway M, O’Brien K, Gelb $A$, et al. The effects of clinical case management on hospital service use among ED frequent users. Am J Emerg Med. 2000;18(5):6003-8.

12. Whittemore R, Knafl K. The Integrative Review: updates methodology. J Adv Nurs. 2005;52(5):546-53.

13. Melnyk BM, Fineout-Overholt E. Making case for evidencebased practice. In: Melnyk BM, Fineou-Overholt E. Evidence based practice in nursing \& healthcare. A guide to practice. Philadelphia: Lippincot Williams \& Wilkins; 2005. p. 324.

14. Xu KT, Nelson BK, Berk S. The changing profile of patients who used Emergency Department Services in United States: 1996 to 2005. Ann Emerg Med. 2009;54(6):805-10. serviços de saúde por usuários frequentes de serviços de urgência para oferecer subsídios para a formulação de políticas públicas de saúde. Destaca-se também a necessidade de discussões sobre esses indivíduos na área da Enfermagem, que atua na assistência e no gerenciamento dos serviços de saúde, sendo indispensável para a ampliação do conhecimento científico sobre a temática e na construção de novas práticas.

15. Olsson M, Hansagi H. Repeted use of emergency department: qualitative study of the patient's perspective. Emerg Med J. 2001;18:430-4.

16. Doup MB, Palatnick W, Day S, Chateau D, Soodeen RA, Burchill C, et al. Frequent Users of Emergency Departments: developing standard definitions and defining prominent risk factors. Ann Emerg Med. 2012 Jul;60(1):24-32.

17. Wajnberg A, Hwang U, Torres L, Yang S. Characteristics of frequent geriatric users of an urban emergency department. J Emerg Med. 2012 Aug;43(2):376-81.

18. Kirby SE, Dennis SM, Jayasinghe UW, Harris MF. Patient related factors in frequent readmissions: the influence of condition, access to services and patient choice. BMC Health Serv Res. 2010;10:216.

19. Paul P, Heng BH, Seow E, Molina J, Tay SY. Predictors of frequent attenders of emergency department Singapore. Emerg Med J. 2010;27(11):843-8.

20. Tangherlini N, Pletcher MJ, Covec MA, Brown JF. Frequent use of emergency medical services by the elderly: a case-control study using paramedic records. Prehosp Disaster Med. 2010;25(3):258-64.

21. Sandoval E, Smith S, Walter J, Schuman SA, Olson MP, Striefler $\mathrm{R}$, et al. A comparison of frequent and infrequent visitors to an urban emergency department. J Emerg Med. 2010; 38(2):115-21.

22. Moore L, Deehan A, Seed P, Jones R. Characteristics of frequent attenders in an Emergency Department: analysis of 1year attendance data. Emerg Med J. 2009; 26:263-267. 23. Aisiku IP, Smith WR, McClish DK, Levenson JL, Penberthy LT, Roseff SD, et al. Comparisons of high versus low Emergency Department utilizers in sickle cell disease. Ann Emerg Med. 2009;53(5):587-93.

24. Milbrett $P$, Halm M. Characteristics and Predictors of Frequent Utilization of Emergency Services. J Emerg Nurs. 2009;35(3):191-8.

25. Huang JA, Weng RH, Lai CS, Hu JS. Exploring medical utilization patterns of Emergency Department users. J Formos Med Assoc. 2008;107(2):119-28.

26. Jelinek GA, Jiwa M, Gibson NP, Lynch AM. Frequent attenders at ED: a linked-data population study of adult patients. Med J Aust. 2008;17;189(10):552-6.

27. Mehl-Madrona LE. Prevalence of psychiatric diagnoses among frequent users of rural emergency medical services. Can J Rural Med.2008;13(1):22-30.

28. Oliveira A. Hiper utilizadores de urgência. Acta Med Port. 2008;21(6):553-8.

29. Fuda KK, Immekus R. Frequent users of Massachusetts Emergency Departments: a statewide analysis. Ann Emerg Med. 2006;48(1):9-16.

30. Blank FSJ, Li H, Henneman PL, Smithline HA, Santoro JS, Provost D, et al. A Descriptive Study of Heavy Emergency Department Users at an Academic Emergency Department 
Reveals Heavy ED Users Have Better Access to Care Than Average Users. J Emerg Nurs, 2005; 31(2):139-44.

31. Torrecillas DR, Rosich AR, Pons MG, Solé MB. Pacientes hiperfrecuentadores en los servicios de urgencias. Estudio descriptivo en un hospital de segundo nivel. Emergencias. 2004;16:178-183.

32. Chan TB, Ovens HJ. Chronic Migraineurs: an important subgroup of patients who visit emergency departments frequently. Ann Emerg Med. 2004;43(2):238-42.

33. Dent AW, Phillips GA, Chenhall AJ, McGregor LR. The heaviest repeat users of an inner city emergency department are not general practice patients. Emerg Med (Fremantle). 2003;15(4):322-9.

34. Byrne M, Murphy AW, Plunkett PK, McGee HM, Murray A, Bury G. Frequent attenders to an emergency department: a study of primary health care use, medical profile, and psychosocial characteristics. Ann Emerg Med. 2003;41(3):30918.

35. Chan TB, Ovens HJ. Frequent Users of Emergency Departments: Do they also use family phisicians' services? Can Fam Physician. 2002;48:1654-60.

36. Hansagi H, Olsson M, Sjöberg S, Tomson Y, Göransson S. Frequent use of the hospital emergency department is indicative of high use of other health care services. Ann Emerg Med. 2001;37(6):561-7.

37. Williams ER, Guthrie E, Mackway-Jones K, James M, Tomenson B, Eastham J, et al. Psychiatric status, somatisation, and health care utilization of frequent attenders at the emergency department. A comparison with routine attenders. J Psychosom Res. 2001;50(3):161-7.

38. Mandelberg JH, Kuhn RE, Kohn MA. Epidemiologic Analysis of an Urban, Public Emergency Department's Frequent Users. Acad Emerg Med, 2000; 7(6):637-46.

39. Locker TE, Baston S, Mason SM, Nicholl J. Defining frequent use of an urban emergency department. Emerg Med J.2007;24(6):398-401.

40. Ruger JP, Richter CJ, Spitznagel EL, Lewis LM. Analysis of costs, length of stay, and utilization of Emergency Department Services by frequent users: implications for health policy. Acad Emerg Med. 2004;11(12):1311-7.

41. Marques GQ, Lima MADS, Ciconet RM. Agravos clínicos atendidos pelo Serviço de Atendimento Móvel de Urgência (SAMU) de Porto Alegre - RS. Acta Paul Enferm. 2011;24(2):18595.

42. Souza SPS, Lima RAG. Chronic condition and normality: towards the movement that broadens the power of acting and being happy. Rev Lat Am Enfermagem. 2007;15(1):156-64. 43. Marques GQ, Lima MADS. Demandas de usuários a um serviço de pronto atendimento e seu acolhimento ao sistema de saúde. Rev Lat Am Enfermagem. [Internet]. 2007 [cited 2012 jan 20];15(1). Available from:

http://www.scielo.br/pdf/rlae/v15n1/pt_v15n1a03.pdf.

Artigo recebido em 15/03/2012.

Aprovado para publicação em 22/08/2012.

Artigo publicado em 30/06/2013. 